\title{
Comparison of the Effectiveness of Ablative and Non-Ablative Fractional Laser Treatments for Early Stage Thyroidectomy Scars
}

\author{
Jin-Uk Jang, Soo-Young Kim, Eul-Sik Yoon, Woo-Kyung Kim, Seung-Ha Park, Byung-Il Lee, \\ Deok-Woo Kim \\ Department of Plastic Surgery, Korea University College of Medicine, Seoul, Korea
}

\begin{abstract}
Background Open thyroidectomy is conventionally performed at the anterior side of neck, which is a body part with a comparatively great degree of open exposure; due to this, postoperative scarring may cause distress in patients. We aimed to compare the effects of ablative and nonablative fractional laser treatments on thyroidectomy scars. We examined medical records in a retrospective manner and analyzed scars based on their digital images by using the modified Manchester Scar Scale (mMSS).

Methods Between February 2012 and May 2013, 55 patients with thyroidectomy scars were treated with ablative (34 patients) or nonablative (21 patients) fractional laser. Each patient underwent 4 laser treatment sessions in 3-4 week intervals, 1-2 months postoperatively. Scar improvement was assessed using patient images and the mMSS scale.

Results The mean decrease in scar score was 3.91 and 3.47 in the ablative and nonablative groups, respectively; the reduction between 2 groups did not exhibit any significant difference $(P=0.16)$. We used the scale once again to individually evaluate scar attributes. The nonablative group accounted for a considerably higher color score value $(P=0.03)$; the ablative group accounted for a considerably higher contour score value $(P<0.01)$. Patient satisfaction was high and no complications occurred.

Conclusions Both types of fractional laser treatments can be used successfully for thyroidectomy scar treatment with minimal complications; however, results indicate that higher effectiveness may be obtained from the use of ablative and nonablative lasers for hypertrophic scars and early erythematous scars, respectively. Therefore, the appropriate laser for scar treatment should be selected according to its specific characteristics.
\end{abstract}

Keywords Laser therapy / Cicatrix / Thyroidectomy

Received: 30 May $2016 \bullet$ Revised: 8 Sep $2016 \bullet$ Accepted: 12 Oct 2016

pISSN: 2234-6163 • elSSN: 2234-6171 • https://doi.org/10.5999/aps.2016.43.6.575• Arch Plast Surg 2016;43:575-581
Correspondence: Eul-Sik Yoon Department of Plastic Surgery, Korea University College of Medicine, 73 Inchon-ro, Seongbuk-gu, Seoul 02841 , Korea

Tel: $+82-2-920-5368$

Fax: +82-2-922-7437

E-mail: yesanam2@korea.ac.kr

Presented at the 30th Annual Meeting of the Korean Society for Laser Medicine and Surgery on November 29, 2015 in Seoul, Korea.

No potential conflict of interest relevant to this article was reported.

\section{INTRODUCTION}

Open thyroidectomies are performed on the prominent and easily exposed anterior neck area; this may leave an unsightly scar, which can be distressing to patients. Thus, proper treatment of a thyroidectomy scar is important, and many attempts at interventions have resulted in the improvement of the clinical appearance of scars to varying degrees of success [1]. Recently, 
strategies using fractional ablative and nonablative lasers have demonstrated safe improvement in the appearance of postoperative, atrophic, and acne scars.

The development of the technique of fractional laser resurfacing based on the principle of fractional photothermolysis (FP) has addressed the limitations of both ablative laser resurfacing and nonablative dermal remodeling, with the respective issues of significant adverse effects and limited efficacy. Similar to the nonablative fractional resurfacing method, ablative fractional resurfacing involves the deposit of a pixilated pattern of microscopic ablated wounds surrounded by a healthy tissue, which may result in both greater efficacy and shorter downtime in relation to FP $[2,3]$. Due to the recent clinical use of nonablative fractional lasers, together with the fact that the stratum corneum is left intact and epidermal barrier function is preserved after treatment, both the extent and severity of side effects and downtime are considerably reduced [2-4]. The use of ablative fractional lasers has also recently increased. Ablative fractional lasers are used for tissue ablation and immediate collagen shrinkage, as well as dermal collagen remodeling, which in turn triggers the removal of collagenous matrix and the synthesis of new collagen by the matrix metalloproteinases $[2,5]$. The reputation of ablative fractional laser treatment is improving thanks to its superiority in energy delivery and better outcomes from fewer treatment sessions.

Nonetheless, few studies have been published comparing the effects of ablative and nonablative fractional laser treatments. Moreover, early scar intervention has been the main focus in recent research, for which many types of lasers have been used for the improvement of scar appearance. Therefore, in this study we have compared the efficacy of ablative and nonablative fractional laser treatments on thyroidectomy scars as part of early scar intervention.

\section{METHODS}

\section{Patients and laser treatments}

This study was conducted with the approval of the Institutional Review Board (IRB No. MD14012). We examined medical records in a retrospective manner and analyzed scars based on their digital images. Between February 2012 and May 2013, 55 patients with thyroidectomy scars were treated using either a nonablative fractional laser (1,550 nm erbium-glass; Mosaic, Lutronic Corp., Goyang, Korea) (21 patients) or an ablative fractional laser $\left(10,600 \mathrm{~nm}\right.$ carbon-dioxide; $\mathrm{eCO}_{2}$, Lutronic Corp.) (34 patients). Thyroidectomy was implemented by the same method (incision and closure) in a linear shape with $7-\mathrm{cm}$ width based on subcutaneous buried suture using Vicryl (4-0,
6-0, Ethicon, Somerville, NJ, USA), and the skin was closed with Dermabond (Ethicon). The mean age was 49.7 and 44.3 years, and the patients had type 3-4 skin according to the Fitzpatrick scale (Table 1). Pregnant or lactating patients, patients receiving concomitant treatment for the skin areas at issue, or patients with keloid scarring experiences were excluded. Each patient received 4 sessions of laser treatment at intervals of 3-4 weeks, beginning 1-2 months after thyroidectomy. To alleviate discomfort, patients were given a 5\% lidocaine cream (EMLA, AstraZeneca, London, UK), 30 minutes prior to treatment, and their faces were cleansed with $0.2 \%$ chlorhexidine gluconate. The laser device and treatment parameters used for each patient were chosen based on scar characteristics. Based on the existing study results and our past experience, the nonablative method was applied in cases where erythema was comparatively severe relative to other parameters, and the ablative method was applied in cases where contour irregularity stood out among the parameters. Treatment parameters ranged from $20-40 \mathrm{~mJ}, 200$ spots $/ \mathrm{cm}^{2}$ density with static operating mode for the nonablative fractional laser, to $100-150 \mathrm{~mJ}, 120-150$ spots $/ \mathrm{cm}^{2}$ density with static operating mode for the ablative fractional laser. The treated areas were cooled after treatment using ice packs for 5-10 minutes. To promote post-laser therapy wound healing, the patients were advised to apply moisturizer on the wound a few times a day for a 1-week period after treatment. All patients provided written informed consent upon enrollment.

\section{Scar assessment}

Digital images of the scars were acquired both prior to and 4 months after treatment. The digital images were taken with the same digital camera (D300, Nikon, Tokyo, Japan), under identi-

Table 1. Demographics and scar characteristics

\begin{tabular}{|c|c|c|c|}
\hline Characteristic & $\begin{array}{c}\text { Ablative } \\
\text { fractional } \\
\text { laser group } \\
(\mathrm{n}=34)\end{array}$ & $\begin{array}{l}\text { Nonablative } \\
\text { fractional } \\
\text { laser group } \\
(n=21)\end{array}$ & P-value ${ }^{a)}$ \\
\hline Age (yr) & $49.7 \pm 11.2$ & $44.3 \pm 11.6$ & 0.62 \\
\hline Sex & & & 0.71 \\
\hline Male & $12(35.3)$ & $6(28.6)$ & \\
\hline Female & $22(64.7)$ & $15(71.4)$ & \\
\hline Type of thyroidectomy & & & 0.59 \\
\hline Total & $22(64.7)$ & $12(57.1)$ & \\
\hline Partial & $12(35.3)$ & $9(42.9)$ & \\
\hline Duration from operation (wk) & $6.5 \pm 1.3$ & $5.9 \pm 1.0$ & 0.66 \\
\hline Scar length $(\mathrm{cm})$ & $7.1 \pm 0.9$ & $7.5 \pm 1.1$ & 0.40 \\
\hline \multicolumn{4}{|l|}{ Pretreatment score } \\
\hline Color & $2.06 \pm 0.72$ & $2.28 \pm 0.65$ & 0.10 \\
\hline Contour & $2.24 \pm 0.53$ & $2.03 \pm 0.53$ & 0.18 \\
\hline
\end{tabular}


cal light source, exposure, background, and white balance. Scar improvement was then assessed using the modified Manchester Scar Scale (mMSS) (discussed below) (Table 2). Adobe Photoshop (Adobe Systems Incorporated, San Jose, CA, USA) was used to measure the $\mathrm{L}^{*} \mathrm{a}^{*} \mathrm{~b}^{*}$ color coordinates of the scar as well as the normal skin surrounding the scar. The $\mathrm{L}^{*} \mathrm{a}^{*} \mathrm{~b}^{*}$ color difference $(\Delta \mathrm{E})$ of the scar and normal surrounding skin was measured by applying the equation provided below [6]:

$$
\Delta \mathrm{E}=\sqrt{\left(\Delta \mathrm{L}^{*}\right)^{2}+\left(\Delta a^{*}\right)^{2}+\left(\Delta b^{*}\right)^{2}}
$$

The $\Delta \mathrm{E}$ values were within the range of $0-32$, with the average value accounting for 8.80 . The $\Delta \mathrm{E}$ value was used for the assessment of mMSS color mismatch (perfect, <6; slight, 6-12; obvious, 12-18; gross, $\leq 18$ ) (Table 2). Based on this scale, possible

\begin{tabular}{|c|c|c|}
\hline Component & & Score \\
\hline Visual analogue scale & Excellent to poor & $1-10$ \\
\hline \multirow[t]{4}{*}{ Color } & Perfect: $\Delta \mathrm{E}^{\mathrm{a})}<6$ & 1 \\
\hline & Slight mismatch: $6 \leq \Delta \mathrm{E}<12$ & 2 \\
\hline & Obvious mismatch: $12 \leq \Delta \mathrm{E}<18$ & 3 \\
\hline & Gross mismatch: $18 \leq \Delta \mathrm{E}$ & 4 \\
\hline \multirow[t]{2}{*}{ Matte or shiny } & Matte & 1 \\
\hline & Shiny & 2 \\
\hline \multirow[t]{4}{*}{ Contour } & Flush with surrounding skin & 1 \\
\hline & Slightly proud/indented & 2 \\
\hline & Hypertrophic & 3 \\
\hline & Keloid & 4 \\
\hline \multirow[t]{4}{*}{ Distortion } & None & 1 \\
\hline & Mild & 2 \\
\hline & Moderate & 3 \\
\hline & Severe & 4 \\
\hline Total & & $4-24$ \\
\hline
\end{tabular}

scores were within the range of 4-24, with higher score values referring to scars with worse conditions. We observed scar improvements based on the changes in the mMSS scores from before and after laser treatments. Three physicians reviewed the visual analogue scale (VAS) score, color, contour, matte/shine, and distortion according to the scale under Table 1 . In case a consensus was not reached, we determined the score based on majority judgment. Scar texture was excluded from the review, as it was impossible to examine this attribute based on a digital image.

\section{Statistical analyses}

Changes in the overall mMSS scores for each individual were analyzed for significance using the paired t-test. While the overall mMSS scores prior to and after each treatment were similar to a normal distribution according to the normality test (Kolmogorov-Smirnov, Shapiro-Wilk test), some of the changes in the respective individual items (volume of reduction) before and after each treatment did not follow normal distribution according to the normality test. Accordingly, the Mann-Whitney $\mathrm{U}$ test, which is a nonparametric test, was used to analyze the decreases in the mMSS, VAS, color, matte/shine, contour, and distortion scores between the 2 groups. A P-value $<0.05$ was considered as statistically significant. We performed all statistical analyses based on SPSS for Windows, ver. 20.0 (IBM Corp., Armonk, NY, USA).

\section{RESULTS}

Both fractional laser treatments led to a decrease in the mMSS, VAS, color, matte/shine, contour, and distortion scores with few complications (Figs. 1-4). The mean initial mMSS scores were $13.26 \pm 2.31$ and $13.14 \pm 2.07$ in the ablative and nonablative

Fig. 1. Ablative fractional laser

A 33-year-old woman had a thyroidectomy scar on her neck. (A) Before treatment, the modified Manchester scar scale score was 13. (B) After 4 sessions of ablative fractional laser treatment (10,600-nm eCO2; Lutronic Corp., Goyang, Korea), the score decreased to 9.
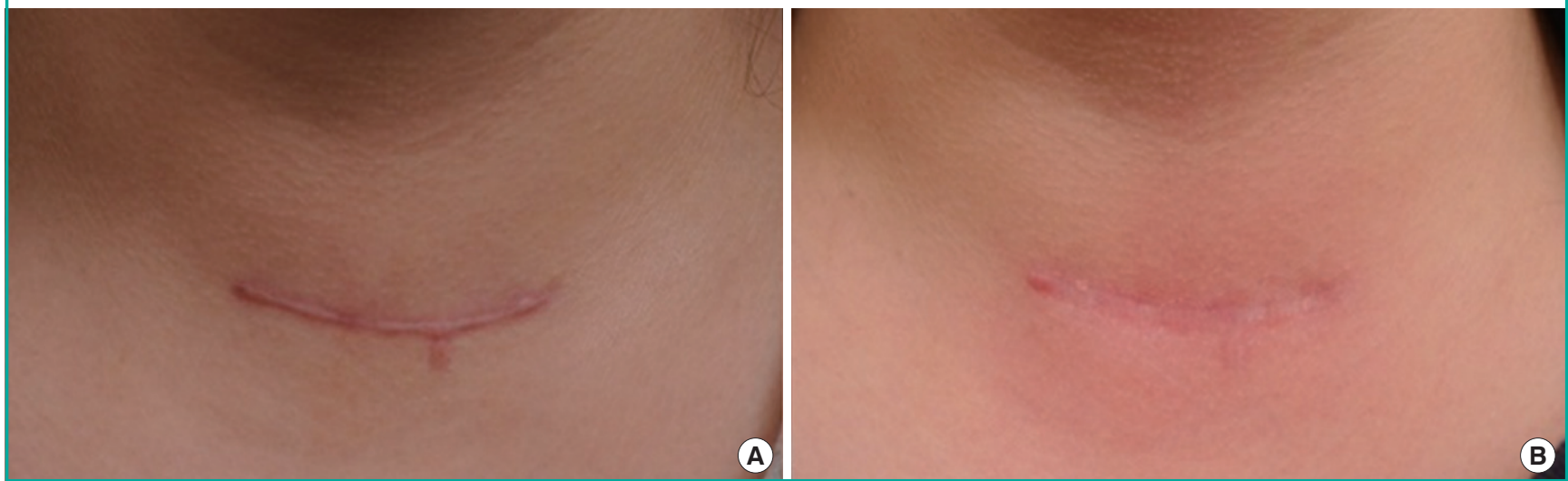


\section{Fig. 2. Nonablative fractional laser}

A 53-year-old woman had a thyroidectomy scar on her neck. (A) Before treatment, the modified Manchester Scar Score was 12. (B) After 4 sessions of non-ablative fractional laser treatment (1,550-nm Mosaic; Lutronic Corp., Goyang, Korea), the score decreased to 9.
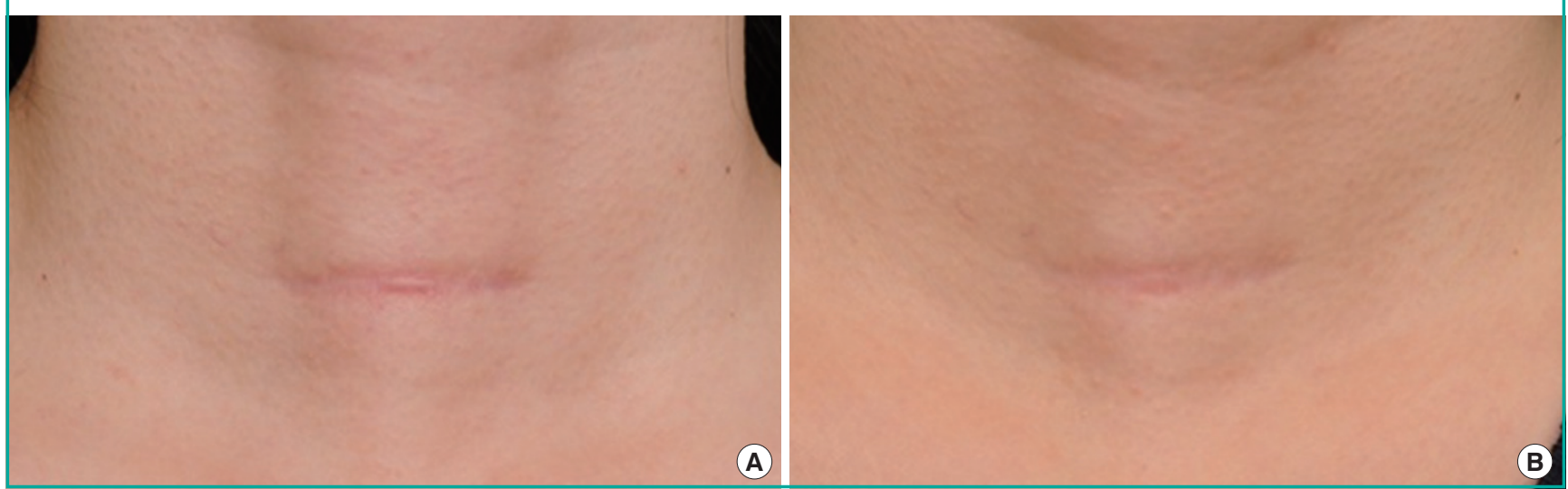

\section{Fig. 3. In the ablative group}

In the ablative group, the total modified Manchester Scar Score (mMSS), the visual analogue scale (VAS) score (5.35 to 3.62), color score (2.68 to 1.97), mattel shine score (1.35 to 1.21$)$, contour score (2.35 to 1.35 ), and distortion score (1.53 to 1.21 ) significantly decreased after treatment.

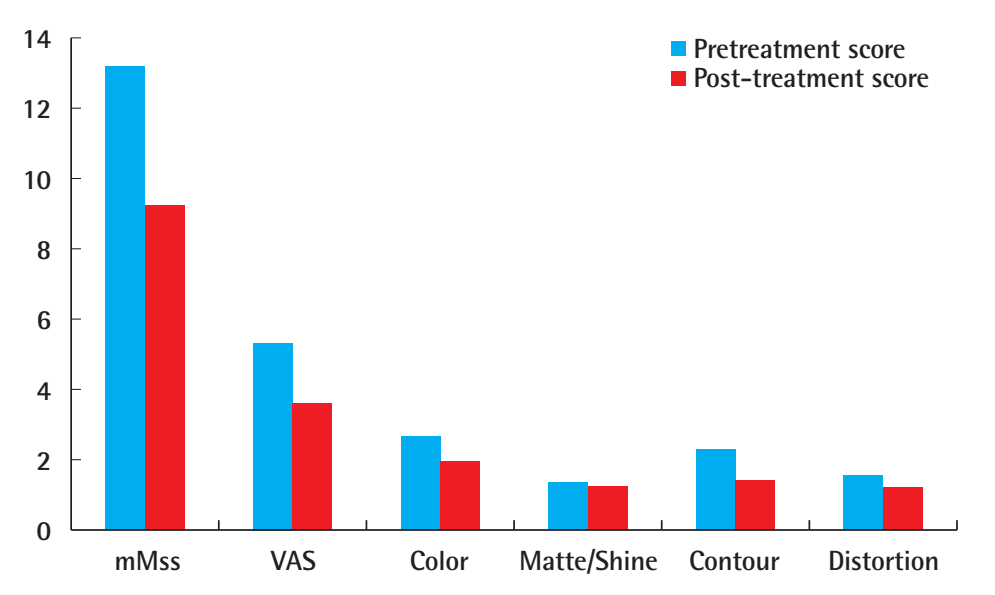

\section{Fig. 4. In the non-ablative group}

In the non-ablative group, the total modified Manchester Scar Score (mMSS), the visual analogue scale (VAS) score (5.43 to 3.76), color score (2.71 to 1.76 ), matte/ shine score (1.52 to 1.33), contour score (2.1 to 1.71), and distortion score (1.38 to 1.1) significantly decreased after treatment.

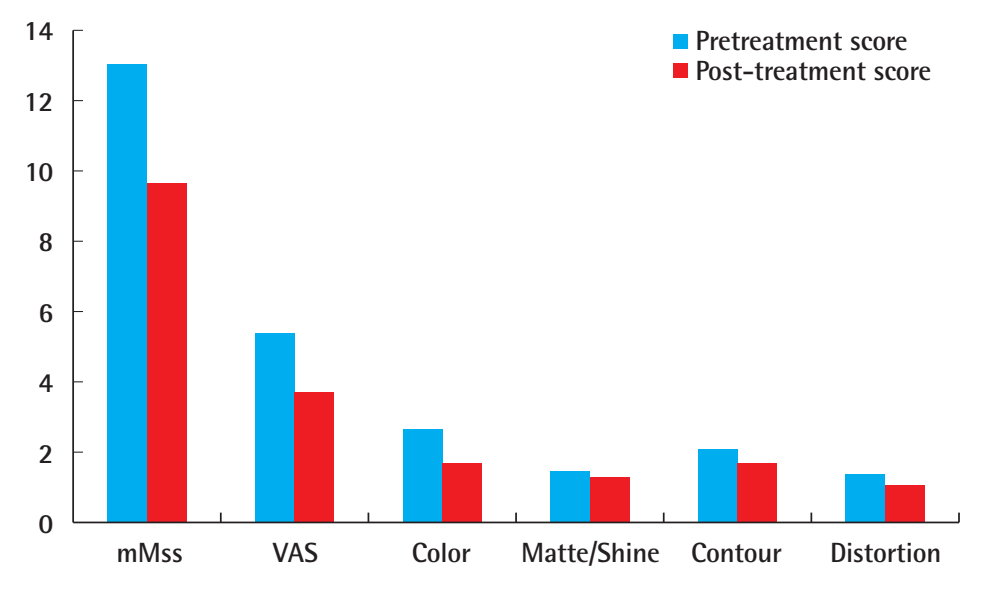

groups, respectively. The initial scores were not significantly different between the 2 groups (independent t-test, $\mathrm{P}=0.84$ ). The mean mMSS scores after laser treatment were $9.35 \pm 2.51$ and
$9.67 \pm 1.45$ in the ablative and nonablative groups, respectively. Both groups showed statistically significant improvements (paired t-test, $\mathrm{P}<0.01$ ) (Table 3 ). The mean decreases in the 
Table 3. Changes in the mMSS score after laser treatment

\begin{tabular}{|lccc|}
\hline mMSS score & $\begin{array}{c}\text { Pretreatment } \\
\text { score }\end{array}$ & $\begin{array}{c}\text { Post- } \\
\text { treatment } \\
\text { score }\end{array}$ & P-value ${ }^{\text {a) }}$ \\
\hline $\begin{array}{l}\text { Ablative fractional laser group } \\
(n=34)\end{array}$ & $13.26 \pm 2.31$ & $9.35 \pm 2.51$ & $<0.01^{*}$ \\
$\begin{array}{l}\text { Non-ablative fractional laser } \\
\text { group ( } n=21)\end{array}$ & $13.14 \pm 2.07$ & $9.67 \pm 1.45$ & $<0.01^{*}$ \\
\hline
\end{tabular}

Values are presented as mean \pm standard deviation.

mMSS, modified Manchester Scar Scale.

a)Paired t-test. ${ }^{*} \mathrm{P}<0.05$

mMSS scores were 3.91 and 3.47 in the ablative and nonablative groups, respectively; the improvement was greater in the ablative group, but this difference did not imply any statistical significance $(P=0.16)$ (Table 4$)$. The scar scale was assessed independently after subdivision considering VAS, color, matte/ shine, contour, and distortion. In the ablative group, the VAS (5.35 to 3.62 ), color (2.68 to 1.97 ), matte/shine (1.35 to 1.21 ), contour (2.35 to 1.35$)$, and distortion (1.53 to 1.21$)$ were significantly lower after treatment (paired t-test, $\mathrm{P}<0.01$ ) (Fig. 3). In addition, in the nonablative group, the VAS (5.43 to 3.76), color (2.71 to 1.76 ), matte/shine (1.52 to 1.33 ), contour (2.1 to 1.71 ), and distortion (1.38 to 1.1 ) were significantly lower after treatment (paired t-test, $\mathrm{P}<0.01$ ) (Fig. 4). In addition to the overall score, the mMSS components (VAS, color, matte/shine, contour, and distortion) were assessed independently, showing score decreases of $2(0-5), 1(0-2), 0(0-1), 1(0-2)$, and 0 (01) (as a mean decrease, $1.74,0.71,0.15,1$, and 0.32 ), respectively, for the ablative group, and 2 (0-3), $1(0-3), 0$ (0-1), 0 (0-1), and $0(0-1)$ (as a mean decrease, 1.67, 0.95, 0.19, 0.38, and 0.29 ), respectively, for the nonablative group (Table 4). The results indicated that the color score value decrease was greater in the nonablative than in the ablative group, which was statistically significant $(\mathrm{P}=0.03)$ (Table 4$)$. In contrast, the ablative group accounted for a greater contour score value decrease than the nonablative group, and these results were also statistically significant $(\mathrm{P}<0.01)$ (Table 4$)$. The 2 groups showed no significant differences in the other values.

\section{DISCUSSION}

A variety of scales exist for the assessment of scars. Nevertheless, a comprehensive scale suitable for all assessment purposes is currently not available. The Vancouver Scar Scale (VSS) is typically used for the assessment of burn scarring. However, the VSS has limitations, as it was designed to review the presence or absence of pathological conditions, without the capability to assess the severity of the scar, making the scale unsuitable for the

\section{Table 4. Decreases in the mMSS}

\begin{tabular}{|lccc|}
\hline $\begin{array}{l}\text { Decrease in the } \\
\text { score }\end{array}$ & $\begin{array}{c}\text { Ablative } \\
\text { fractional laser } \\
\text { group } \\
(\mathbf{n = 3 4 )}\end{array}$ & $\begin{array}{c}\text { Nonablative } \\
\text { fractional laser } \\
\text { group } \\
(\mathbf{n = 2 1 )}\end{array}$ & P-value ${ }^{\text {a) }}$ \\
\hline mMSS & $4(0-7)$ & $4(1-5)$ & 0.16 \\
Visual analogue scale & $2(0-5)$ & $2(0-3)$ & 0.72 \\
Color & $1(0-2)$ & $1(0-3)$ & $0.03^{*}$ \\
Matte/shine & $0(0-1)$ & $0(0-1)$ & 0.67 \\
Contour & $1(0-2)$ & $0(0-1)$ & $<0.01^{*}$ \\
Distortion & $0(0-1)$ & $0(0-1)$ & 0.77 \\
\hline $\begin{array}{l}\text { Values are presented as median (interquartile range). } \\
\text { mMSS, modified Manchester Scar Scale. }\end{array}$ & \\
alMann-Whitney U test. *P ${ }^{*}<0.05$. & & \\
\hline
\end{tabular}

assessment of linear scars [7]. The mMSS is another type of scale commonly used for both linear and hypertrophic scars that assesses the VAS score, color, contour, matte/shine, texture, and distortion. In this study, we applied the mMSS to scar images; the image scores were in line with and highly correlated to the clinical score of the respective scars, with the texture feature being the exception [8].

A surgical scar is a common cosmetic problem, but treatment for this outcome is complicated. It is known that aesthetic disfigurement, as a consequence of scarring, may lead to psychological debilitation, not only impairing quality of life but also overall productivity in the subject patients [9]. Scar formation is often influenced by dermatological traits related to factors such as race, genetic traits, and other individual factors. Therefore, prediction of hypertrophic scar and keloid formation is difficult at the time of the surgery, making prevention even more difficult. Although a variety of treatments are available for scar correction after surgery, full restoration of the sites of alreadyformed mature scars to the original state is impossible. Application of cream, ointment or silicone patches, pressure therapy, and steroid injection have been used as diverse intervention methods against the formation of hypertrophic scar, with their efficacy still under debate [10].

Laser treatment gained popularity in the 1980s following the description of selective FP by Anderson and Parrish [11], which was used for skin rejuvenation not long afterwards. Ablative laser skin resurfacing is one of the methods of facial rejuvenation treatment renowned for its surprisingly satisfactory effects [12]. Alster and Zaulyanov [9] demonstrated that skin resurfacing with ablative lasers has proven to be an effective option for scar treatment. Despite generally good results, ablative laser treatment also has limitations, one of which is the adverse effects relating to a long postoperative period before patients can resume social activities [13]. A complete epidermal ablation leads to a long period of social withdrawal, long-lasting erythema, changes 
in skin pigmentation, as well as scarring and possible delay in healing [14]. Meanwhile, a previous study evaluated the efficacy and safety of long-pulse 1,320-nm neodymium-doped yttrium aluminium garnet and 1,450-nm diode lasers in the treatment of atrophic facial scarring [15]. Those authors reported that, within the scope of the study, the 1,450 -nm diode laser resulted in better clinical scar response. Thus, they concluded that nonablative laser treatment is a satisfactory alternative for atrophic scarring patients for whom the long postoperative recovery procedure associated with ablative laser skin resurfacing would pose difficulties.

Fractional lasers, which were designed to overcome the limitations mentioned above, are gaining popularity in this regard. In 2004, Manstein et al.s [2] description of FP offered the possibility of treating photoaging with fewer risks and reduced downtime. The fractioning feature of the laser beams enabled the preservation of healthy skin area, leading to an acceleration in the healing procedure [16]. Fractional lasers create countless microthermal zones, consisting of a main necrotizing zone and its neighboring thermal effect zones, where the healthy skin maintains its barrier function, which helps the acceleration of the healing procedure [2]. Fractional lasers are well known for the treatment of a variety of scar types, with recent studies showing that early scar treatment based on the use of fractional lasers leads to aesthetic satisfaction [17].

In 2007, Hantash et al. conducted a study on ablative fractional lasers, which, theoretically, were better for dermal remodeling compared to other nonablative lasers. Due to less tissue damage, a number of nonablative laser sessions are necessary for the comparison of their efficacy with that of a single session of ablative laser treatment. Similar results were reached using the fractional $\mathrm{CO}_{2}$ laser. Chapas et al. [18] discovered that after 2-3 treatment sessions, 13 patients with facial atrophic acne scarring saw an average of $66.8 \%$ improvement. Choe et al. [19] found that the formation of hypertrophic scarring could be successfully prevented with fractional laser treatment in patients who underwent total thyroidectomy. Another previous study reported that ablative fractional laser treatment may be superior to nonablative fractional laser treatment in improving the appearance and texture of scars, especially in reducing the visibility of the incision line [20]. Recently, scar treatment with both nonablative FP by a 1,550-nm erbium-glass fractional laser system, and with ablation by a $10,600-\mathrm{nm} \mathrm{CO}_{2}$ fractional laser system, has been reported to be safe and tolerable, producing minimal adverse effects and providing acceptable clinical improvement [21].

This study has several advantages. First, we compared the effects of ablative and nonablative fractional lasers on uniform scars (where the same institution performed the same type of treatment on the same scar location). Based on such a method, we were able to prevent potential errors that may occur from differences in scar location, suture method, etc. Second, this study was conducted based on a larger pool of cases compared to existing studies, and used the mMSS, an appropriate scar assessment tool. In particular, this tool is the most adequate among scar assessment tools for use in retrospective studies. This study also has some limitations. First, since the patient pool was selected based on scar characteristics instead of randomized control, selection bias is likely to exist from a statistical standpoint. However, based on a retrospective statistical comparison of the pretreatment scores of the 2 patient pools regarding color and contour item, which the authors judged to be statistically meaningful, the 2 pools did not exhibit any essential difference, and therefore it was considered reasonable to maintain the conclusion (pretreatment score in Table 1). Second, while the scar remodeling phase typically requires a long-term observation period (over 18 months), the follow-up observation ended earlier than the typical period, and consequently the study did not include the observation of natural improvement followed by a lapse of time. The lack of a control group is another factor that may need to be addressed in further research.

We found that both ablative and nonablative fractional laser treatment significantly improved thyroidectomy scars without significant complications. In particular, with regard to the components of the mMSS, ablative fractional laser treatment was more effective than nonablative fractional laser treatment in improving contour. In contrast, nonablative fractional laser treatment was superior to ablative fractional laser treatment in improving color. Thus, ablative fractional laser treatment appears to offer greater efficiency in the treatment of hypertrophic scars, whereas nonablative fractional laser treatment may be useful for early erythematous scars. These results are in agreement with those of studies conducted to date. Therefore, the laser device for scar treatment should be chosen according to scar characteristics. To date no laser-related parameter for thyroidectomy scar prevention, such as high energy, low density, or low energy with high density, has been established. It is essential to continue accumulating clinical data to further optimize laser treatment strategies for thyroidectomy scars and other scarring.

\section{REFERENCES}

1. Conologue TD, Norwood C. Treatment of surgical scars with the cryogen-cooled $595 \mathrm{~nm}$ pulsed dye laser starting on the day of suture removal. Dermatol Surg 2006;32:13-20.

2. Manstein D, Herron GS, Sink RK, et al. Fractional photo- 
thermolysis: a new concept for cutaneous remodeling using microscopic patterns of thermal injury. Lasers Surg Med 2004;34:426-38.

3. Geronemus RG. Fractional photothermolysis: current and future applications. Lasers Surg Med 2006;38:169-76.

4. Laubach HJ, Tannous Z, Anderson RR, et al. Skin responses to fractional photothermolysis. Lasers Surg Med 2006;38: 142-9.

5. Fisher GJ, Varani J, Voorhees JJ. Looking older: fibroblast collapse and therapeutic implications. Arch Dermatol 2008; 144:666-72.

6. Cheon YW, Lee WJ, Rah DK. Objective and quantitative evaluation of scar color using the $\mathrm{L}^{*} \mathrm{a}^{*} \mathrm{~b}^{*}$ color coordinates. J Craniofac Surg 2010;21:679-84.

7. van der Wal MB, Verhaegen PD, Middelkoop E, et al. A clinimetric overview of scar assessment scales. J Burn Care Res 2012;33:e79-87.

8. Beausang E, Floyd H, Dunn KW, et al. A new quantitative scale for clinical scar assessment. Plast Reconstr Surg 1998; 102:1954-61.

9. Alster T, Zaulyanov L. Laser scar revision: a review. Dermatol Surg 2007;33:131-40.

10. Alster TS, Tanzi EL. Hypertrophic scars and keloids: etiology and management. Am J Clin Dermatol 2003;4:235-43.

11. Anderson RR, Parrish JA. Selective photothermolysis: precise microsurgery by selective absorption of pulsed radiation. Science 1983;220:524-7.

12. Rosenberg GJ, Brito MA Jr, Aportella R, et al. Long-term histologic effects of the CO2 laser. Plast Reconstr Surg 1999; 104:2239-44.

13. Houk LD, Humphreys T. Masers to magic bullets: an updated history of lasers in dermatology. Clin Dermatol 2007;25:
434-42.

14. Nanni CA, Alster TS. Complications of carbon dioxide laser resurfacing: an evaluation of 500 patients. Dermatol Surg 1998;24:315-20.

15. Tanzi EL, Alster TS. Comparison of a 1450-nm diode laser and a 1320-nm Nd:YAG laser in the treatment of atrophic facial scars: a prospective clinical and histologic study. Dermatol Surg 2004;30:152-7.

16. Borges J, Cuzzi T, Mandarim-de-Lacerda CA, et al. Fractional Erbium laser in the treatment of photoaging: randomized comparative, clinical and histopathological study of ablative $(2940 \mathrm{~nm})$ vs. non-ablative $(1540 \mathrm{~nm})$ methods after 3 months. An Bras Dermatol 2014;89:250-8.

17. Kim SG, Kim EY, Kim YJ, et al. The efficacy and safety of ablative fractional resurfacing using a 2,940-Nm Er:YAG laser for traumatic scars in the early posttraumatic period. Arch Plast Surg 2012;39:232-7.

18. Chapas AM, Brightman L, Sukal S, et al. Successful treatment of acneiform scarring with $\mathrm{CO} 2$ ablative fractional resurfacing. Lasers Surg Med 2008;40:381-6.

19. Choe JH, Park YL, Kim BJ, et al. Prevention of thyroidectomy scar using a new 1,550-nm fractional erbium-glass laser. Dermatol Surg 2009;35:1199-205.

20. Kim HS, Lee JH, Park YM, et al. Comparison of the effectiveness of nonablative fractional laser versus ablative fractional laser in thyroidectomy scar prevention: a pilot study. J Cosmet Laser Ther 2012;14:89-93.

21. Cho S, Jung JY, Shin JU, et al. Non-ablative $1550 \mathrm{~nm}$ erbium-glass and ablative 10,600 $\mathrm{nm}$ carbon dioxide fractional lasers for various types of scars in Asian people: evaluation of 100 patients. Photomed Laser Surg 2014;32:42-6. 